\title{
Improving the quality of wool through the use of gene markers
}

\author{
T.O. Itenge ${ }^{1 \#}$, J.G.H. Hickford ${ }^{2}$, R.H.J. Forrest ${ }^{2}$, G.W. McKenzie ${ }^{2}$ and C.M. Frampton ${ }^{3}$ \\ ${ }^{1}$ Department of Animal Science, Faculty of Agriculture and Natural Resources, University of Namibia, Private Bag \\ 13301, Windhoek, Namibia \\ ${ }^{2}$ Department of Agricultural Sciences, Facility of Agriculture and Life Sciences, PO Box 84, Lincoln University, \\ Christchurch 7647, New Zealand \\ ${ }^{3}$ University of Otago, Christchurch School of Medicine and Health Sciences, P.O. Box 4345, Christchurch 8140, \\ New Zealand
}

\begin{abstract}
This study aimed at identifying gene markers associated with wool quality traits in Merino and Merino Cross sheep using a candidate gene approach. Polymerase chain reaction-single strand conformational polymorphism (PCR-SSCP) analysis was used to identify sequence variation in the KAP1.3 and K33 genes, while agarose gel electrophoresis was used to detect length variation in the KAP1.1 gene. Two half-sib families (SL1 and SL2) were created for analysis and wool samples were collected from the mid-side region of the SL1 progeny at 12, 24 and 36 months of age, and of the SL2 progeny at 12 months of age. The association of alleles was analysed for each year of phenotypic data by an analysis of variance (ANOVA) tests, using SPSS ${ }^{\circledR}$ version 15. Analysis of each of KAP1.1, KAP1.3 and K33 genes revealed potential gene markers to select for animals with increased staple length, increased staple strength, higher yield, whiter and brighter wool. The results obtained are consistent with KAP1.1, KAP1.3 and KRT1.2 being clustered on one chromosome. Results also indicated that the keratin genes on chromosome 11 are recombining relatively frequently at recombination "hotspots". It appears as though genes coding for the KRTs and KAPs have the potential to impact on wool quality and could potentially be exploited in gene marker-assisted selection programmes in the wool industry for the rapid conversion of wool from one type to another
\end{abstract}

Keywords: Sheep, wool, keratin, gene marker, allele, KAP, KRT

${ }^{\#}$ Corresponding author. E-mail: titenge@unam.na

\section{Introduction}

The efficiency of wool processing is dependent on the consistency of wool fibre. Although selective breeding of sheep has in part reduced the variation in wool fibres, considerable variation both within and between fleeces still exists. The use of genetic markers would greatly accelerate the efficiency of wool breeding programmes.

The keratin intermediate-filament proteins (KRTs) and keratin intermediate-filament associated proteins (KAPs) are the major proteins that make up the wool fibre. The KRTs form the skeletal structure of the wool fibre (microfibrils) and are embedded in a matrix of KAPs (Powell \& Rogers, 1986). These proteins are connected through disulphide cross-linkages which are important for the stability and the mechanical properties of wool (Feughelman, 1997).

Numerous studies have described variation within both the KAP and KRT genes, including the work of Rogers et al. (1994a); Parsons et al. (1994a; 1996); McLaren et al. (1997) and Beh et al. (2001). This genetic diversity could impact on wool fibre structure and hence variation in the genes, and proteins derived from them could therefore underpin variation in wool traits.

In this respect there are reports associating variation in the KRT and KAP genes with variation in wool traits. Parsons et al. (1994b) and Beh et al. (2001) reported associations between variation in mean fibre diameter and KAPs in Merino sheep, while Rogers et al. (1994b) reported a putative QTL association between staple strength in Romney sheep and the region spanning the KAP1.1 (formally B2A), KAP1.3 (formally B2C) and K33 (formally KRT1.2) loci, on ovine chromosome 11.

The aim of this study was to identify variation in the KAP1.1, KAP1.3, and K33 genes, and to determine whether variation in the three genes is associated with variation in wool traits within two Merino half-sib families, referred to as Sire Line 1 (SL1) and Sire Line 2 (SL2). 


\section{Materials and Methods}

The study used two half-sib families. The SL1 half-sib was created by mating a fine wool producer Merino ram to 150 Merino ewes, selected at random from a range of New Zealand environments in order to maximise phenotypic variation in wool traits. In total, 169 lambs were born. However, some died at or after birth, so that 131 lambs survived during the first year and were used in this study. Of these, only 128 survived to the second shearing in the second year. Subsequent to the second shearing the wethers were culled and only the remaining ewe lambs $(\mathrm{n}=37)$ were shorn in the third year.

The second half-sib family was created by mating the two-tooth ewes from the SL1 half-sib to a halfbred (Merino X Corriedale) ram. Forty-three lambs were born, and 35 lambs survived and were used in the study. Half-sib groups were kept as single flocks to minimise environmental variation between individual progeny. All lambs were tagged at birth and DNA from the lambs, ewes and sires was collected onto FTA cards (Whatman, Middlesex, UK), and processed using the manufacturer's protocol.

The KAP1.1 locus was genotyped using amplified fragment length polymorphism (AFLP) and agarose gel electrophoresis, while the KAP1.3 and K33 loci were genotyped using polymerase chain reactions-single strand conformational polymorphism (PCR-SSCP). The amplification and genotyping protocols used are described in Itenge-Mweza et al. (2007). For the KAP1.3 locus a second set of PCR-SSCP conditions were also employed as it was difficult to distinguish between the $C$ and $I$ allele banding patterns and the $A$ and $J$ (GenBank accession FJ263959) allele banding patterns. These conditions differed to those described in Itenge-Mweza et al. (2007) in that the primers used for the PCR were upstream 5'CAAGCAGACCAAACTCAGAAAC-3' and downstream 5'-TGTCCACAGTAGGATGGGCGGC-3' (defining a 436 bp amplimer) and the polyacrylamide gel used for the SSCP analysis contained 1\% glycerol instead of $0.2 \%$. All other conditions remained the same as described in Itenge-Mweza et al. (2007).

Mid-side wool samples were collected at 12, 24 and 36 months of age for SL1 and at 12 months of age for SL2. Except for greasy fleece weight (GFW) which was determined at shearing, wool measurement was performed by the New Zealand Wool Testing Authority Ltd (NZWTA, Napier, New Zealand) according to International Wool Textile Organisation (IWTO) standards. Measurements included comfort factor, mean fibre diameter (MFD, IWTO-12-03), fibre diameter standard deviation (FDSD, IWTO-12-03), coefficient of variation of fibre diameter (CVD, IWTO-12-03) and curvature were measured using the Sirelan-Laserscan Fibre Diameter analyser while the mean staple length (MSL, IWTO-30) and mean staple strength (MSS, IWTO-30) of each sample were determined using ATLAS (Automatic tester for Length and Strength). The colour (MY-Z) and brightness (MB) of the wool were measured using a reflectance spectrophotometer, where the tristimulus values $\mathrm{Y}-\mathrm{Z}$ indicate the yellowness of the wool and the tristimulus value $\mathrm{Y}$ represents the brightness of the wool. The yield of wool, the weight of clean wool after impurities such as vegetable matter have been removed, expressed as a percentage of greasy wool weight can be mathematically derived from the wool base (IWTO-19) measurements. Once yield measurements were obtained from the NZWTA, clean fleece weight (CFW) was calculated as the product of GFW and yield. Colour traits MY-Z and MB were not measured in SL1 in the second year and CFW and GFW were not measured in SL1 in years 2 and 3 or in the SL2 half-sib.

All analyses were performed using SPSS ${ }^{\circledR}$ version 15 (SPSS Science Inc., Chicago, IL, USA). For each trait measured, an ANOVA was performed which included gender and birth type (either single or twins) as factors to test the effect of gender and birth type on each trait. For each sire line and each locus (KAP1.1, KAP1.3 and K33), half-sib segregation analyses were performed to test the association of paternally inherited alleles with all measured wool traits. Any progeny that typed the same as the sire and dam were removed from the data set as the paternally inherited allele could not be determined. If gender and/or birth rank were previously found to have an effect $(\mathrm{P}<0.10)$ on a particular trait, they were included as factors in the General Linear Models used to test the effect of paternally inherited alleles on that trait. Otherwise t-tests were used, with groups being split by paternal allele.

\section{Results and Discussion}

Three alleles (designated A-C, GenBank accession AY835603-AY835605, respectively) that varied in length were observed at the KAP1.1 locus. Although single nucleotide substitutions were observed between the KAP1.1 alleles, the major difference are that the $A$ allele encodes one more decapeptide repeat than the $B$ allele and two repeats more than the $C$ allele (Rogers et al., 1994a). Nine alleles (designated A-I, GenBank 
accession AY835589 - AY83596 and FJ263959, respectively) were identified at the KAP1.3 locus and five alleles (designated A-E, GenBank accession numbers AY835598-AY835602) were identified at the K33 locus. Sequence analysis of the KAP1.3 and K33 alleles revealed that all of the variation resulted from predominantly single nucleotide substitutions. The genotype of SL1 progeny at the KAP1.1, KAP1.3 and $\mathrm{K} 33$ loci was $A B, B D$ and $A B$, respectively, while that of SL2 was $B C, C J$ and $D E$, respectively.

Gender was found to have a significant effect on wool yield in both sire lines. In SL1, 24 months of age rams had a mean yield of $76.9 \pm 0.40 \%$ which was higher $(\mathrm{P}=0.013)$ than the ewes which had a mean yield of $75.5 \pm 0.40 \%$. In SL2, rams also had a higher yield $(\mathrm{P}=0.015)$ than ewes, with rams having a mean yield of $73.2 \pm 1.43 \%$ while ewes had a mean yield of $69.8 \pm 1.74 \%$. No other gender effects were detected for any of the traits measured (data not shown).

Birth rank was found to impact on comfort factor $(\mathrm{F}>30, \mathrm{P}<0.001)$, MFD $(\mathrm{P}=0.001)$, MSL $(\mathrm{P}=$ 0.011), MB ( $\mathrm{P}=0.030)$, GFW $(\mathrm{P}<0.001)$ and CFW $(\mathrm{P}<0.001)$ in $\mathrm{SL1}$ progeny but was restricted to samples taken at 12 months of age. No other birth rank effects were detected for any of the traits measured (data not shown). The progeny of SL2 were all singles except one set of twins.

Analysis of each of the informative loci revealed allelic associations with various wool traits. In SL1, KAP1.1 $A$ allele was associated with a higher yield $(76.86 \pm 0.451 \%)$ at 24 months of age than $B$ allele (75.5 \pm $0.43 \%)(\mathrm{P}=0.037)$, with a similar trend showing at 36 months, $A$ allele $(79.1 \pm 1.10 \%)$ and $B$ allele $(76.7 \pm$ $0.83 \%)(P=0.078)$, but not at 12 months. In the SL2, the KAP1.1 $B$ allele was associated with increased staple length. It is of note that in the SL1, variation at the KAP1.1 locus also showed a strong trend $(\mathrm{P}=$ 0.080 ) with staple length at 12 months of age. Since the KAP1.1 A allele encodes one more decapeptide repeat than the $B$ allele, it could be argued that KAP1.1 allelic variation might impact on yield and wool length because the decapeptide repeat unit contains two cysteine residues and is therefore more "costly" to the sheep, especially as it has been shown previously that increased dietary intake of cysteine or methionine can increase wool growth (Hemsley \& Reis, 1985). However, the higher MSL (78.2 $\pm 4.0 \mathrm{~mm})$ associated with the KAP1.1 $B$ allele in SL2 in this study compared to the $C$ allele $(70.5 \pm 8.6 \mathrm{~mm})$, would not support this hypothesis.

Allelic variation in KAP1.3 was found to be significantly associated with variation in MY-Z in SL1, and MSL and MB in SL2. In SL1, allele $D$ tended to be associated with higher yield when compared to progeny that inherited allele $B$ at 24 months of age. The major difference between KAP1.3 alleles $B$ and $D$ (accession numbers AY835590 and AY835592, respectively) is the presence of a single nucleotide difference, $337 \mathrm{G} \rightarrow \mathrm{A}$ in the $\mathrm{D}$ allele. This substitution was silent, although recently it has been shown in an unrelated mammalian protein that a single nucleotide polymorphism can affect the timing of co-translational folding, thereby altering the structure of the protein (Kimchi-Sarfaty et al., 2006).

Allelic variation in K33 was found to be significantly associated with FDSD1, MSS1 and MY-Z3, in SL1 and MSL and MB in SL2. K33 allele $B$ was also associated with variation in staple strength at 12 months of age in SL1. The association was not observed at 24 or 36 months of age. However, power analyses (results not shown) suggested that there were inadequate numbers of SL1 progeny numbers to detect a $10 \%$ difference between sire allele groups for staple strength at 24 and 36 months of age, and therefore it is possible that in the presence of a larger sample size, an association may have been observed. Results by Rogers et al. (1994b) showed evidence of a QTL for wool strength on chromosome 11 in the region of the KAP1.1 (B2A), KAP1.3 (B2C) and K33 (KRT1.2) loci in Romney sheep. The finding from this study is therefore in agreement with the Rogers et al. (1994b) findings, but it is only a single observation and it has not been seen in SL2 half-sib family. The difference observed in the staple strength between the progeny inheriting K33 $A$ and $B$ alleles could be commercially significant, as at low MFD there is emerging evidence that wool price is driven as much by MSS as MFD.

Both sires passed on major KAP1.1 - KAP1.3-KRT1.2 haplotypes to their progeny. The two major haplotypes inherited from Sire Line 1 were BBA (frequency of $43.4 \% ; n=43$ ) and ADB (frequency of $44.4 \% ; \mathrm{n}=44$ ), and from Sire Line 2 were CCE (frequency of $53.1 \% ; \mathrm{n}=17$ ) and BJD (frequency of $40.6 \%$; $\mathrm{n}=13$ ). In order to account for haplotype effects, a comparison was performed between wool from progeny that inherited one major haplotype to progeny that inherited the other major haplotype from their sire and the progeny with allele recombinations were removed from the analysis, and similarity of the trait averages was observed. 
The results obtained suggest that KAP1.1, KAP1.3 and KRT1.2 are tightly linked because both sires in the study passed on two major haplotypes to their progeny, and the similarity of the trait averages also supports the close linkage between these keratin loci that has previously been reported by Rogers et al. (1994b) and McLaren et al. (1997). Minor haplotypes were also observed, giving an overall recombination rate of at least $14 \%$ in SL1, and 6.3\% in SL2. This suggests a relatively high rate of recombination between the loci. Tightly linked loci do not usually recombine, and if they do, they have a low recombination rate. There was therefore some evidence to suggest that keratin genes on chromosome 11 are recombining very frequently, and it is speculated that this recombination occurs at recombination hotspots.

Within SL1 it might have been expected that an allele that was associated with a trait in a particular year, would be associated with the same trait in the other two years. However, this was not always the case. A possible explanation for this may be that there are differences in the protein composition of wool from older sheep compared to lambs. There may also be effects on wool traits that act in an age-dependent manner. The possibility of wool traits being affected by other environmental factors also cannot be discounted. Across the lines the sires had different alleles and these alleles arguably could have different effects on the traits. Accordingly SL1 might have two alleles that result in an unresolvable phenotypic difference while SL2 may not. For all the genes studied, that is KAP1.1, KAP1.3 and KRT1.2, the sires in the two lines had different genotypes, so no between sire comparison could easily be drawn.

\section{Conclusion}

A large amount of genetic variation was revealed at the keratin gene loci, despite the fact that only two sire lines of Merino origin were studied. Other sheep breeds are likely to reveal even greater allelic variation. These findings provide further support for the important role of the KAP and KRT genes in determining variation wool fibre characteristics, although considerably more research will be required to understand the full impact of the variation seen in these genes. It appears as though genes coding for the KRTs and KAPs could potentially be exploited in gene marker-assisted selection programmes to produce wool better suited to consumer demands.

\section{Acknowledgment}

We wish to thank the New Zealand Wool Testing Authority (NZWTA) and JBT Consulting Ltd for the financial support of this research.

\section{References}

Beh, K.J., Callaghan, M.J., Leish, Z., Hulme, D.J., Lenane, I. \& Maddox, J.F., 2001. A genome scan for QTL affecting fleece and wool traits in Merino sheep. Wool Technology and Sheep Breeding.49, 8889.

Feughelman, M., 1996. Mechanical properties and structure of alpha-keratin fibres: wool, human hair and related fibres. UNSW Press Sydney, Australia.

Hemsley, J.A. \& Reis, P.J., 1985. Amino acids and wool growth. In: Ruminant physiology - concepts and consequences. Proceedings of a symposium held at the University of Western Australia. Eds Baker, S.K., Gawthorne, J.M., Mackintosh, J.B. \& Purser, D.B., University of Western Australia, Australia. pp. 253-261.

Itenge-Mweza, T.O., Forrest, R.H.J., McKenzie, G.W., Hogan, A., Abbott, J., Amoafo, O. \& Hickford, J.G.H., 2007. Polymorphism of the KAP1.1, KAP1.3 and K33 genes in Merino sheep. Molecular and Cellular Probes 21, 338-342.

Kimchi-Sarfaty, C., Oh, J. M., Kim, I-W., Sauna, Z. E., Calcagno, A. M., Ambudkar, S.V. and Gottesman, M. M., 2006. A "silent" polymorphism in the MDR1 gene changes substrate specificity. Science 315, 525-528.

McLaren, R.J., Rogers, G.R., Davies, K.P., Maddox, J.F. \& Montgomery, G. W., 1997. Linkage mapping of wool keratin and keratin-associated protein genes in sheep. Mamm. Genome 8, 938-940.

Parsons, Y.M., Cooper, D.W. \& Piper, L.R., 1996. Genetic variation in Australian Merino sheep. Anim. Genet. 27, 223-228.

Parsons, Y.M., Piper, L.R., Powell, B.C. \& Cooper, D.W., 1994a. Wool keratin gene polymorphisms and production characters in Australian Merino. Anim. Genet. 21, 113-116. 
Parsons, Y.M., Cooper, D.W. \& Piper, L.R., 1994b. Evidence of linkage between high-glycine-tyrosine keratin gene loci and wool fibre diameter in a merino half-sib family. Animal Genetics. 25:105-108.

Powell, B.C. and Rogers, G. E., 1986. Hair keratin: composition, structure and biogenesis, Volume 2. In: Biology of the Integument. (Edited by Bereiter-Hahn J., Matoltsy A. G. and Richards K. S.), pp. 695721. Springer-Verlag, Berlin.

Rogers, G.R., Hickford, J.G.H. \& Bickerstaffe, R., 1994a. Polymorphism in two genes for B2 high sulphur proteins of wool. Anim. Genet. 25, 407-415.

Rogers, G.R., Hickford, J.G.H. \& Bickerstaffe, R., 1994b. A potential QTL for wool strength located on ovine chromosome 11. Proceedings, $5^{\text {th }}$ World Congress on Genetics Applied to Livestock Production, University of Guelph, Guelph, Ontario, Canada. 21, 291-294. 